\title{
Research on Intelligence of AGC System in Hot Strip Mill
}

\author{
Xibang Zhang ${ }^{1,}{ }^{*}$, Fengqin Wang ${ }^{1}$, Ziying Liu ${ }^{1}$, JianXin Fan² ${ }^{2}$ Xiaobin Huang ${ }^{2}$ \\ ${ }^{1}$ Process Technology Department, Shougang Research Institute of Technology, Beijing 100043, \\ China;
}

${ }^{2}$ Hot Metal Department, Shougang Qian'an Iron and Steel Company, Qian'an 064404, Hebei, China. *ustbzxb@163.com

Keywords: Thickness control; rolling force age; monitor age; age function optimization.

\begin{abstract}
The thickness control system is an important method to realize the high precision hot rolling. By analyzing the Siemens thickness control model, this paper introduces the algorithm and principle of MN-AGC with smith predictor. The SIEMENS CFC program was used for fuzzy MN-AGC function development, to solve the problem that the original Siemens AGC gain coefficient is single. After application, the strip thickness control precision has been further improved.
\end{abstract}

\section{Introduction}

Thickness accuracy is the most important indicator of hot rolled strip. There are various disturbances resulting in thickness deviation in rolling process, so the automatic thickness control system is necessary [1]. The AGC model guarantees the quality of rolling products and the rolling speed and represents the core technology of the steel industry. The AGC model of major steel mills is almost entirely developed and commissioned by foreigners, among which German Siemens and Japan TMEIC (Toshiba Mitsubishi) are represented. Such as the German Siemens SIMATIC TDC system, and Japan TMEIC Corporation V3000 System [2].

This paper introduces the algorithm of Siemens thickness control model for hot strip mill. According to the thickness problem, develop AGC intelligent control function.

\section{Siemens Thickness Control Model}

\subsection{Double-Ring Control System.}

Siemens thickness control system uses a typical double-ring series system, the inner ring is the position closed loop system APC, the outer ring is the thickness closed loop system AGC, and the two rings are connected by the integral link [3]. The double-loop AGC is shown as Figure 1.

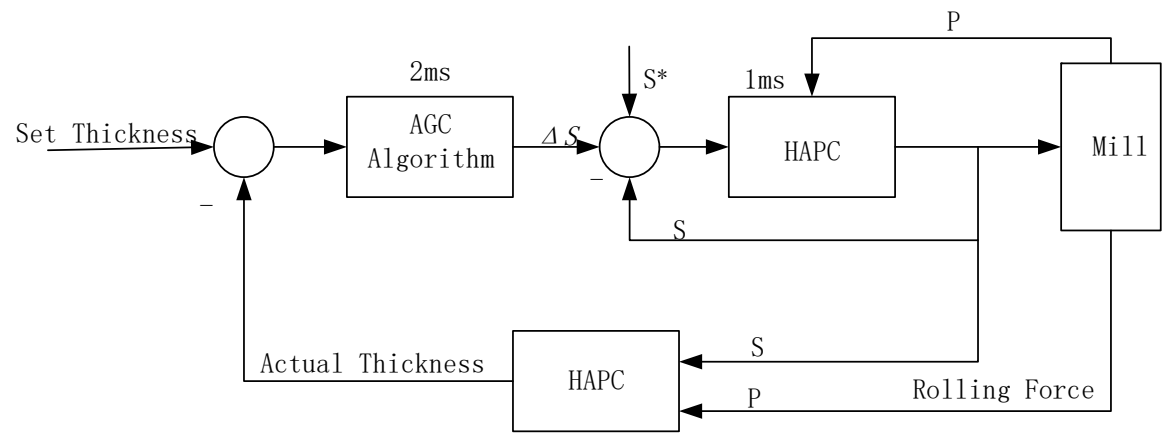

Figure. 1. Siemens double-loop AGC control system diagram

The Siemens thickness control system consists of the rolling force AGC (RF-AGC) and the monitoring AGC (MN-AGC). Which F1 F3 stands use RF-AGC, and F4 F6 stands use two control methods of RF-AGC and MN-AGC. The thickness control system consists of two kinds of AGC mode such as absolute AGC and relative AGC. The default is to use the absolute AGC method to adjust. If the actual thickness of the head exceeds the limited thickness, or the actual rolling force of 
the head and the set rolling force deviation is too large, the AGC mode will switch to relative AGC mode.

\subsection{Smith Estimates Strategy for MN-AGC.}

The RF-AGC is "indirect thickness measurement." Although various compensation factors are considered, the thickness calculation accuracy is always lower than the thickness value directly measured by the X-ray thickness measurement. Therefore, the RF-AGC must be used together with the MN-AGC.

The control principle of the MN-AGC is: The exit thickness is directly measured by the thickness gauge installed at the outlet side of the mill. The thickness deviation between the measured thickness and the target thickness is fed back to the thickness automatic control device. The control signal is changed to the roll gap adjustment amount and sent to the APC. So that the AGC system can eliminate thickness deviation [4]. The diagram of MN-AGC control principle is shown in Figure 2.

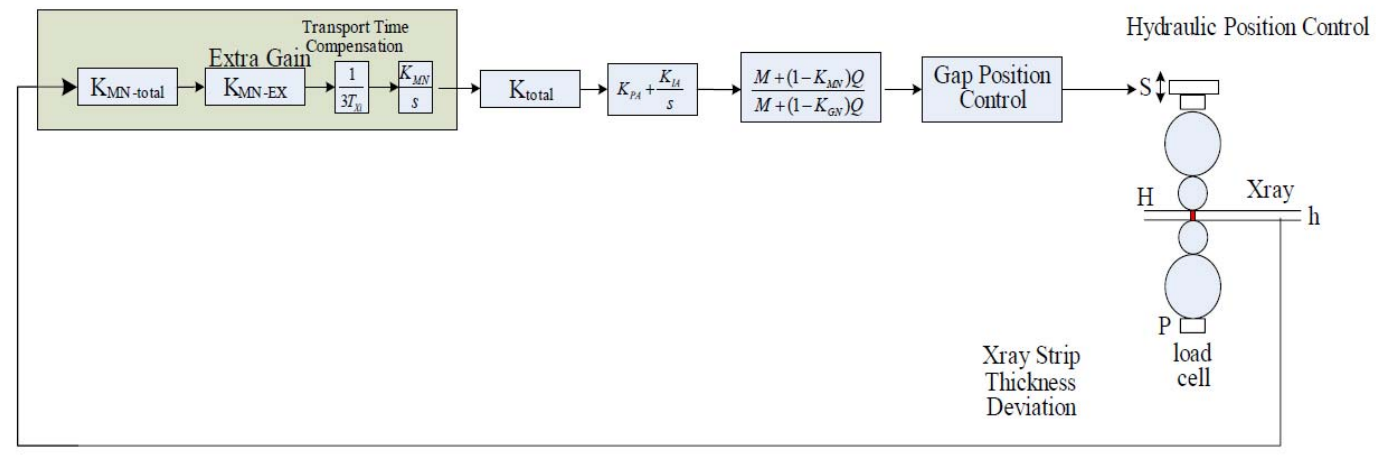

Figure. 2. The diagram of MN-AGC control principle

The expression formula for MN-AGC roll gap adjustment amount is as follows:

$$
\Delta h_{M O N(i)}=K_{M N-A G C} \times \Delta h_{X-R A Y} \times \frac{T_{0 i} \times G_{i}}{T_{d i}} \times K_{p}\left(1+\frac{1}{T_{I} S}\right)
$$

Where $\Delta h_{X-R A Y}$ is the thickness deviation; $T_{0 i}$ correction time constant of strip travel time from stand $\mathrm{i}$ to thickness gauge; $T_{d i}$ the actual time from stand $\mathrm{i}$ to thickness gauge; $G_{i}$ compensation gain of strip travel time from stand $i$ to thickness gauge; $K_{p}\left(1+\frac{1}{T_{I} S}\right)$ PI session.

The thickness gauge is installed at $3.72 \mathrm{~m}$ from the outlet of the final stand. The detection of the actual thickness of the strip and the control of the roll gap adjustment amount do not take place at the same time. To avoid a pure lag in the operation of the MN-AGC system, Siemens AGC model uses the Smith predictor [5]. The predictor model structure is shown in Figure 3.

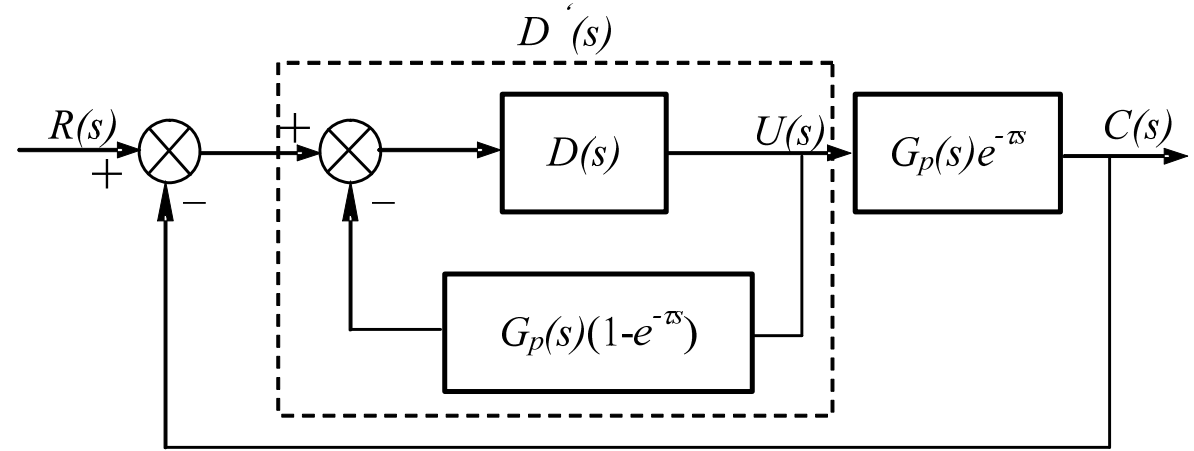

Figure. 3. The diagram of MN-AGC with Smith predictor

The calculated thickness is compared with the thickness detected by the thickness meter and the obtained difference $\mathrm{E}=\mathrm{C}-\mathrm{C}^{\prime}$ is used as the second feedback signal, which is fed back to the AGC to correct the calculated thickness and improve the control accuracy. It is MN-AGC's Smith estimates strategy as the Figure 4 shows. 


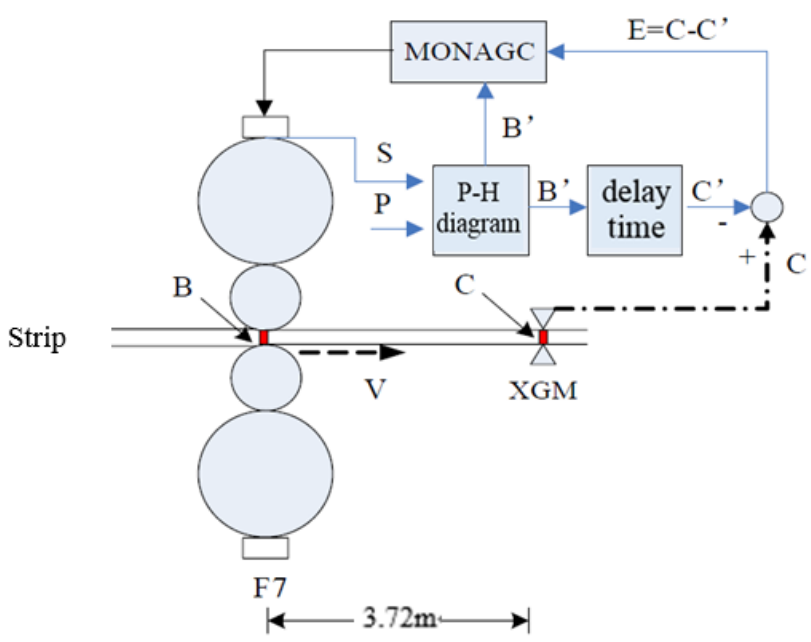

Figure. 4. Strategy of Smith estimates

\section{Fuzzy MN-AGC Function Development}

\subsection{The Description of Thickness Oscillation.}

The basic model of MN-AGC is an integral regulator. The larger the gain coefficient $K_{M N-A G C}$, the faster the adjustment speed. In the Siemens thickness control model, the MN-AGC gain coefficient is set to be single. If the gain coefficient is set large and the thickness detection deviation is small, the AGC overshoot phenomenon tends to occur, which causes the thickness oscillation. On the contrary, if the gain coefficient is set small and the thickness detection deviation is large, the adjustment speed is often not enough to cause the thickness to be adjusted in place in time, resulting in an excessive thickness. As shown in Figure 5 and Figure 6.

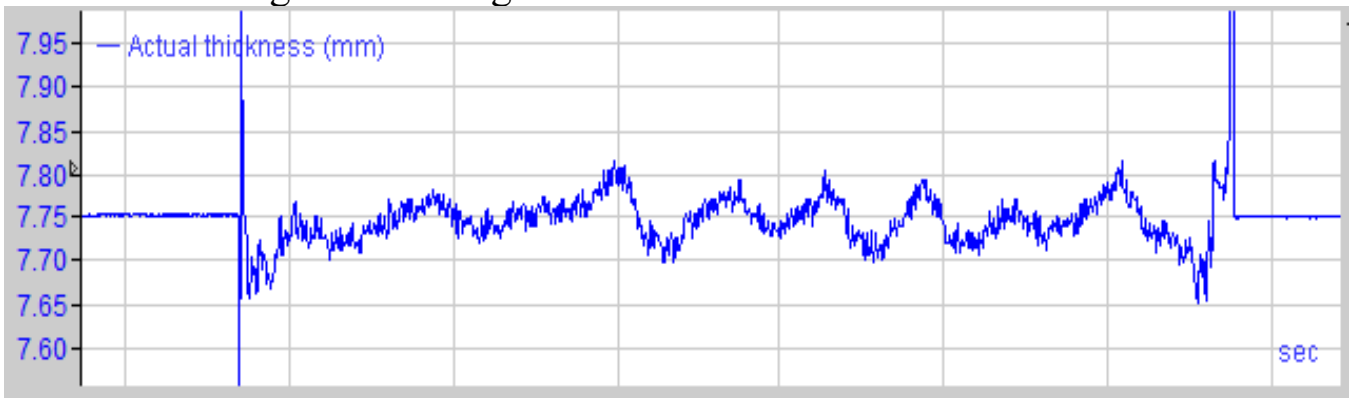

Figure. 5. The problem of thickness oscillation

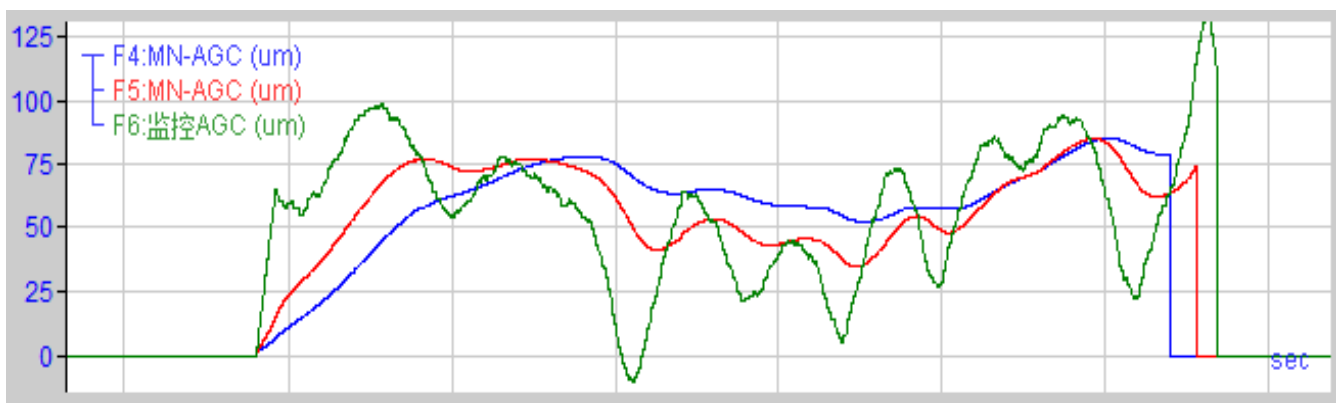

Figure. 6. The problem of MN-AGC adjustment

The thicker the strip, the more obvious the body oscillating phenomenon, and the lower the thickness hit rate. Especially when the thickness is $>=6 \mathrm{~mm}$, the thickness hit rate drops sharply.

\subsection{Fuzzy Controller Applied in MN-AGC.}

The PID control algorithm that MN-AGC single gain coefficient will not meet the requirements [6]. The fuzzy controller is just good at solving this problem. The most important aspect of fuzzy control is to determine the fuzzy control rules, which can be obtained through expert experience, or through sampling data. The fuzzy controller structure and decision table are as follows. 
Table. 1. Monitor AGC fuzzy decision

\begin{tabular}{|c|c|c|c|c|c|c|}
\hline $\begin{array}{c}\Delta h / \mathrm{um} \\
h / \mathrm{mm}\end{array}$ & -100 & -50 & -20 & 20 & 50 & 100 \\
\hline 3 & $0.85 * \mathrm{~A}$ & $0.85 * \mathrm{~B}$ & $0.85^{*} \mathrm{C}$ & $0.85 * \mathrm{C}$ & $0.85 * \mathrm{~B}$ & $0.85 * \mathrm{~A}$ \\
\hline 5 & $0.95 * \mathrm{~A}$ & $0.95 * \mathrm{~B}$ & $0.95^{*} \mathrm{C}$ & $0.95 * \mathrm{C}$ & $0.95 * \mathrm{~B}$ & $0.95 * \mathrm{~A}$ \\
\hline 7 & $\mathrm{~A}$ & $\mathrm{~B}$ & $\mathrm{C}$ & $\mathrm{C}$ & $\mathrm{B}$ & $\mathrm{A}$ \\
\hline 10 & $1.1 * \mathrm{~A}$ & $1.1 * \mathrm{~B}$ & $1.1 * \mathrm{C}$ & $1.1 * \mathrm{C}$ & $1.1 * \mathrm{~B}$ & $1.1 * \mathrm{~A}$ \\
\hline
\end{tabular}

The fuzzy controller applied in MN-AGC, can provide different gain coefficients according Strip thickness specification and actual thickness deviation. Then the thickness deviation amount is large, the rapid adjustment, and the thickness deviation amount is small, the adjustment is slowly. This fuzzy function can prevent the adjustment amount from being insufficient or overshoot, effectively improving the stability of the thickness control, and reducing the thickness overshoot and oscillation.

\section{Conclusion}

By analyzing the Siemens thickness control model, the control principle of the monitoring AGC and the calculation formula of the roll gap adjustment amount are given. By using the Smith predictor, calculate the thickness on this basis and correct it to improve the accuracy of AGC control and the adaptability of AGC to the product.

For the problem that the original Siemens AGC gain coefficient is single, the CFC structured programming is used to develop the fuzzy MN-AGC function, which improves the stability of the thickness control and reduces the thickness overshoot and oscillation.

\section{References}

[1]. X. Zhou and Y. Gao, Thickness Control of Hot Rolling Process at Meagan, Boatel Meehan, 2009, 33-34.

[2]. W. Wang, Design of Automation Gauge Control in Hot Mill of Boatel, Shanghai, Shanghai Jiao Tong University, 2011.

[3]. Z. X. Li and X. H. Liu, Research of the Gauge Control System for 2160 Hot Strip Mill at Shrugging, Metallurgical Industry Automation, 2010, 100-102.

[4]. X. C. Zhang, Development and Application of AGC Control System for $1500 \mathrm{~mm}$ Hot Continuous Rolling in Lagging, Information Technology, 2015, 80-83.

[5]. F. Zhang and Y. J. Zhang, Thickness Control Strategies of Plate Rolling Mill, International Journal of Innovative Computing, 2015, 1227-1230.

[6]. C. N. Tong and Y. K. Sun, Intelligent Control of Hot Continuous Rolling Comprehensive AGC System, University of Science Technology Beijing, 2002, 552-556. 